Annals of Pure and Applied Mathematics

Vol. 17, No. 1, 2018, 35-41

ISSN: 2279-087X (P), 2279-0888(online)

Published on 20 April 2018

Annals of

www.researchmathsci.org

DOI: http://dx.doi.org/10.22457/apam.v17n1a5

Pure and Applied

Mathematics

\title{
Glivenko Congruence on a Nearlattice Related to Semi
} Prime Ideals

\author{
Md. Zaidur Rahman ${ }^{1}$, Momtaz Begum ${ }^{2}$ and A.S.A.Noor ${ }^{2}$ \\ Department of Mathematics \\ Khulna University of Engineering \& Technology \\ E mail: mzrahman1968@gmail.com \\ ${ }^{2}$ Department of CSE, Prime University, Mirpur-1, Dhaka \\ E mail: momoislam81@yahoo.com; asanoor100@gmail.com
}

Received 16 March 2018; accepted 19 April 2018

Abstract. In this paper authors have proved some results on Glivenko congruence $R$ with respect to Semi prime ideal $J$ in a nearlattice $S$. They showed that the quotient nearlattice $\frac{S}{R}$ is distributive if and only if $J$ is semiprime. Moreover, they have included a prime separation theorem for semiprime ideals. At the end some results on $A^{\perp}$ and $A^{0}$ for a 0-distributive nearlattice are given. Finally they have included a characterization of distributive nearlattices with the help of Separation theorems by using semiprime ideals.

Keywords: 0-distributive nearlattice, Semi prime ideal, Glivenko congruence, Quotient nearlattice.

AMS Mathematics Subject Classification (2010): 06A12, 06A99, 06B10

\section{Introduction}

Varlet [8] first introduced the concept of 0-distributive lattices. Then many authors [2, 6] studied them for lattices and semilattices. On the other hand, [4] studied the 0-distributive directed above meet semilattices extensively and discussed different properties of these semilattices by a number of characterizations. Recently $[9,10]$ have studied them for nearlattices. Again [5] have proved several interesting results on 0-distributive nearlattices.

A nearlattice is a meet semilattice together with the property that any two elements possessing a common upper bound have a supremum. This property is known as the upper bound property.

By [9], a nearlattice $S$ with 0 is called 0-distributive if for all $a, b, c \in S$ with $a \wedge b=0=a \wedge c$ imply $a \wedge(b \vee c)=0$ whenever $b \vee c$ exists. [7] Introduced the concept of semiprime ideals in a lattice. Then [1] studied these ideals elaborately and established many interesting results. They also extend the Prime Separation Theorem for 0-distributive lattices, which give a flavour of Separation Theorem for non-distributive 
Md. Zaidur Rahman, Momtaz Begum and A.S.A.Noor

lattices. Then [11] extended the concept for nearlattices. An ideal $J$ of a nearlattice $S$ is called a semiprime ideal if for all $a, b, c \in S$ with $a \wedge b \in J$ and $a \wedge c \in J$ imply $a \wedge(b \vee c) \in J$ whenever $b \vee c$ exists. Hence a nearlattice $S$ with 0 is called 0 distributive if (0] is a semiprime ideal of $S$. Let $A \subseteq S$ and $J$ be an ideal of $S$. We define $A^{\perp_{J}}=\{x \in S: x \wedge a \in J$ for all $a \in A\}$. This is clearly a down set containing $J$. By [11, Theorem 5], we know that when $J$ is semiprime then $A^{\perp_{J}}$ is in fact a semiprime ideal. $A^{\perp_{J}}$ called an annihilator of $A$ relative to $J$.

For an ideal $J$ of a nearlattice $S$, define a relation $\theta$ on $S$ by $a \equiv b(\theta)$ if and only if $(a]^{\perp_{J}}=(b]^{\perp_{J}}$. In otherwords, $a \equiv b(\theta)$ is equivalent to " for each $x \in S$, $a \wedge x \in J$ if and only if $b \wedge x \in J . "$

In this paper, we will show that this is a congruence on the nearlattice $S$ when $J$ is semiprime. We call it as Glivenko congruence. Recently Glivenko congruence have been studied by [12]. In this paper, we extend several results of [12] and then establish some new results.

\section{Main result}

Proposition 2.1. Let $J$ be a semiprime ideal in a nearlattice $S$. Define a relation $R$ on $S$ by $x \equiv y(R)$ if and only if $\{x\}^{\perp_{J}}=\{y\}^{\perp_{J}}$. Then $R$ is a nearlattice congruence on $S$.

Proof: Clearly $R$ is an equivalence relation on $S$. Now let $x \equiv y(R)$ and $t \in S$. Then $\{x\}^{\perp_{J}}=\{y\}^{\perp_{J}}$. Suppose $a \in\{x \wedge t\}^{\perp_{J}}$. Then $a \wedge x \wedge t \in J$ which implies $a \wedge t \in\{x\}^{\perp_{J}}=\{y\}^{\perp_{J}}$. Thus, $a \wedge y \wedge t \in J$ and so $a \in\{y \wedge t\}^{\perp_{J}}$. Therefore $\{x \wedge t\}^{\perp^{J}} \subseteq\{y \wedge t\}^{\perp J}$. Similarly, $\{y \wedge t\}^{\perp^{J}} \subseteq\{x \wedge t\}^{\perp^{J}}$ and so $\{x \wedge t\}^{\perp J}=\{y \wedge t\}^{\perp J}$. Hence $x \wedge t \equiv y \wedge t(R)$. Now let $x \equiv y(R)$ and $x \vee t, \quad y \vee t$ exist for some $t \in S$. Let $a \in\{x \vee t\}^{\perp_{J}}$. Then $a \wedge(x \vee t) \in J$ and so $a \wedge x, a \wedge t \in J$. This implies $a \wedge y, a \wedge t \in J$ as $\{x\}^{\perp_{J}}=\{y\}^{\perp_{J}}$. Therefore $a \wedge(y \vee t) \in J$ as $J$ is semiprime. It follows that $R$ is a nearlattice congruence on $S$.

Remarks: Let $S$ be a nearlattice and $\Theta$ a congruence on $S$. We denote the quotient nearlattice of $S$ modulo $\Theta$ by $\frac{S}{\Theta}$. If $\frac{S}{\Theta}$ has a zero element [0], then [0] is called the kernel of $\Theta$. Clearly $[0]$ is then an ideal of $S$. Notice that we do not require $S$ itself to have a zero element. If $J$ is an ideal of $S$, we shall say that $J$ is the kernel of a homomorphism if there exists a congruence $\Theta$ on $S$ such that $J$ is the kernel of $\Theta$. Thus an ideal $J$ is a kernel provided $J$ is a complete congruence class for some 
Glivenko Congruence on a Nearlattice Related to Semi Prime Ideals congruence $\Theta$ on $S$. Then for every $x \in S$ and any $j \in J, x \geq x \wedge j$ implies $\frac{[x]}{\Theta} \geq J$ in $\frac{S}{\Theta}$. Hence $J$ is the zero element of $\frac{S}{\Theta}$.

Theorem 2.2. Let $S$ be a nearlattice and $J$ be an ideal of $S$. Then the following conditions are equivalent.

(i) $J$ is semiprime.

(ii) $J$ is the kernel of some homomorphism of $\mathrm{S}$ onto a distributive neralattice with 0 .

(iii) $J$ is the kernel of some homomorphism of $\mathrm{S}$ onto a 0-distributive nearlattice.

Proof: $(i) \Rightarrow(i i)$ Consider the elements $[x],[y],[z]$ in $\frac{S}{R}$ such that $y \vee z$ exists where $R$ is the Glivenko congruence. Let $s \equiv x \wedge(y \vee z)(R)$. Then $\{s\}^{\perp J}=\{x \wedge(y \vee z)\}^{\perp_{J}}$. Suppose $t \in\{s\}^{\perp J}$. Then $t \wedge(x \wedge(y \vee z)) \in J$, hence $t \wedge x \in\{y \vee z\}^{\perp_{J}}=\{y\}^{\perp_{J}} \cap\{z\}^{\perp_{J}}$.

Therefore, $t \in\{x \wedge y\}^{\perp_{J}} \cap\{x \wedge z\}^{\perp_{J}}=\{(x \wedge y) \vee(x \wedge z)\}^{\perp_{J}}$. Thus $\{s\}^{\perp_{J}} \subseteq\{(x \wedge y) \vee(x \wedge z)\}^{\perp_{J}}$, equivalently, $\frac{[s]}{R} \leq \frac{[(x \wedge y) \vee(x \wedge z)]}{R}$, hence $\frac{[x]}{R} \wedge\left(\frac{[y]}{R} \vee \frac{[z]}{R}\right) \leq\left(\frac{[x]}{R} \wedge \frac{[y]}{R}\right) \vee\left(\frac{[x]}{R} \wedge \frac{[z]}{R}\right)$. Since the reverse inequality is trivial, so $\frac{S}{R}$ is a distributive nearlattice.

Furthermore, for any $i, j \in J$ clearly $i \equiv j(R)$. Moreover, for any $i \in J$, $i \equiv a(R)$ implies $\{a\}^{\perp_{J}}=\{i\}^{\perp_{J}}=S$. This implies $a \in J$. Thus $J$ is a complete congruence class modulo $R$. That is, $J$ is the kernel of $R$ and so ( $i i)$ holds.

(ii) $\Rightarrow$ (iii) By (ii) $J=k e r \Theta$ for some congruence $\Theta$ on $S$ and $\frac{S}{\Theta}$ is a distributive nearlattice. Since every distributive nearlattice $S$ with 0 is 0 -distributive, so $\frac{S}{\Theta}$ is $\quad$ 0-distributive and so ( $\left.i i i\right)$ holds.

(iii) $\Rightarrow(i)$ Let $\Theta$ be a congruence on $S$ for which $J$ is the zero element of the 0-distributve nearlattice $\frac{S}{\Theta}$. Let $x \wedge y \in J$ and $x \wedge z \in J$ such that $y \vee z$ exists. This implies $\frac{[x]}{\Theta} \wedge \frac{[y]}{\Theta}=\frac{[x \wedge y]}{\Theta}=J=\frac{[y \wedge z]}{\Theta}=[y] \wedge[z]$. Since $\frac{S}{\Theta}$ is 0 -distributive, it 
Md. Zaidur Rahman, Momtaz Begum and A.S.A.Noor

follows that $\frac{[x]}{\Theta} \wedge \frac{([y] \vee[z])}{\Theta}=J$. That is, $\frac{[x \wedge(y \vee z)]}{\Theta}=J$ and so $x \wedge(y \vee z) \in J$. Therefore $J$ is semiprime.

Now we give a separation theorem for semiprime ideals.

Theorem 2.3. Let $J$ be a semiprime ideal of a nearlattice $S$ and $F$ be a filter of $S$ disjoint to $J$. Then there exists a prime ideal $P \supseteq J$ such that $P \cap F=\phi$.

Proof: Define a relation $R$ on $S$ by $x \equiv y(R)$ if and only if $\{x\}^{\perp_{J}}=\{y\}^{\perp_{J}}$. Then by Proposition 2.1 and Theorem 2.2, $R$ is a nearlattice congruence and the quotient nearlattice $\frac{S}{R}$ is distributive. Since $F \cap J=\phi$, so $\frac{F}{R}$ is a proper filter of $\frac{S}{R}$. It follows now from the prime separation theorem for distributive nearlattice [3] that there exist a prime ideal $\frac{P}{R}$ of $\frac{S}{R}$ disjoint to $\frac{F}{R}$. Then clearly, $P=h^{-1}\left(\frac{P}{R}\right)$ is a prime ideal of $S$ containing $J$ and disjoint from $F$, where $h$ is the canonical homomorphism of $S$ onto $\frac{S}{\Theta}$.

By [11, Theorem 5] we know that for any subset $A$ of a 0 -distributive nearlattice $S$, $A^{\perp}=\{x \in S \mid x \wedge a=0$ for all $a \in A\}$ is a semiprime ideal. We also define $A^{0}=\{x \in S \mid x \wedge a=0$ for some $a \in A\}$.

Lemma 2.4. For a meet subsemilattice $A$ of a 0 -distributive nearlattice $S, A^{0}$ is a semiprime ideal.

Proof: By [10, Theorem5], $A^{0}$ is an ideal. Now let $x \wedge y \in A^{0}$ and $x \wedge z \in A^{0}$ for some $x, y, z \in S$ with $y \vee z$ exists. Then $x \wedge y \wedge a=0$ and $x \wedge z \wedge b=0$ for some $a, b \in A$. Since $A$ is a meet subsemilattice, so $a \wedge b \in A$.

Now $x \wedge y \wedge a \wedge b=0=x \wedge z \wedge a \wedge b$ imply $x \wedge a \wedge b \wedge(y \vee z)=0$ as $S$ is a 0 distributive nearlattice. Thus $x \wedge(y \vee z) \in A^{0}$ and so $A^{0}$ is semiprime.

Thus we have the following corollaries.

Corollary 2.5. Let $A$ be a non-empty subset and $F$ be a filter of a 0-distributive nearlattice $S$ such that $A^{\perp} \cap F=\phi$. Then there exists a prime ideal $P$ containing $A^{\perp}$ such that $P \cap F=\phi$. 
Glivenko Congruence on a Nearlattice Related to Semi Prime Ideals

Corollary 2.6. Let $A$ be a meet subsemilattice and $F$ be a filter of a 0-distributive nearlattice $S$ such that $A^{0} \cap F=\phi$ Then there exists a prime ideal $P$ containing $A^{0}$ and $P \cap F=\phi$.

Theorem 2.7. Let $J$ be a semiprime ideal of a nearlattice $S$ and suppose that for some $a, b \in S, a \wedge b \in J$. Then there exist semiprime ideals $A$ and $B$ (possibly improper) such that $a \in A, b \in B$ and $J=A \cap B$.

Proof: If $a \in J$, then by choosing $A=J$ and $B=S$, the theorem trivially holds. So assume hence forth that neither $a$ nor $b$ is in $J$. Now define the relation $R$ on $S$ by $x \equiv y(R)$ if and only if $\{x\}^{\perp_{J}}=\{y\}^{\perp_{J}}$. Since $J$ is semiprime, so by theorem 2.2, $R$ is a nearlattice congruence and $\frac{S}{R}$ is a distributive nearlattice. Let $h: S \rightarrow \frac{S}{R}$ be the canonical homomorphism with kernel $J$. Put $S^{\prime}=\frac{S}{R}$. Thus $S^{\prime}$ is a distributive nearlattice with $0^{\prime}=J$. Hence $a^{\prime} \wedge b^{\prime}=0^{\prime}$, where $a^{\prime}=h(a)$ and $b^{\prime}=h(b)$. By hypothesis $a \notin J, b \notin J$, hence $a^{\prime} \neq 0^{\prime} \neq b^{\prime}$. Choose the ideals $A^{\prime}=\left(a^{\prime} \vee b^{\prime}\right] \cap\left(b^{\prime}\right]^{*}$ and $B^{\prime}=\left(a^{\prime} \vee b^{\prime}\right] \cap\left(a^{\prime}\right]^{*}$ in $\frac{S}{R}$. Since $a^{\prime} \wedge b^{\prime}=0^{\prime}$ it follows that $a^{\prime} \in A^{\prime}$ and $b^{\prime} \in B^{\prime}$.

Clearly $A^{\prime} \cap B^{\prime}=\left(0^{\prime}\right]$. Putting $A=h^{-1} A^{\prime}$ and $B=h^{-1} B^{\prime}$ yields the semiprime ideals $A$ and $B$.

Theorem 2.8. A nearlattice $S$ is distributive if and only if for every ideal $I$ and a filter $F$ of $S$ for which $I \cap F=\phi$ there exists a semiprime ideal $J \supseteq I$ such that $J \cap F=\phi$.

Proof: Suppose $S$ is distributive. Then by [3, Theorem 2.6], there exists a prime ideal $P \supseteq I$ such that $P \cap F=\phi$. Since every prime ideal is semiprime, so choosing $J=P$ we get the result.

Conversely, suppose the condition holds. If $S$ is not distributive. Then there exist $x, y, z \in S$ with $y \vee z$ exists such that $x \wedge(y \vee z)>(x \wedge y) \vee(x \wedge z)$. Consider $I=((x \wedge y) \vee(x \wedge z)]$ and $F=[x \wedge(y \vee z))$. Clearly $I \cap F=\phi$. Then by the given condition, there exists a semiprime ideal $J \supseteq I$ such that $J \cap F=\phi$. Now $x \wedge y \in J$ and $x \wedge z \in J$. Since $J$ is semiprime, so $x \wedge(y \vee z) \in J$. This implies $J \cap F \neq \phi$, which gives a contradiction. Hence $S$ must be distributive.

Finally we include the following Separation Theorem.

Theorem 2.9. Let $F$ be a proper filter of a near lattice $S$ with 0 . Then the following conditions are equivalent.

(i) $\quad S$ is 0-distributive. 
Md. Zaidur Rahman, Momtaz Begum and A.S.A.Noor

(ii) For each finite subset $A$ of $S$ with $F \cap A^{\perp}=\phi$, there exists prime filter

$$
Q \supseteq F \text { such that } Q \cap A^{\perp}=\phi \text {. }
$$

Proof: $(i) \Rightarrow(i i)$. Since $\mathrm{S}$ is 0 -distributive, so by [11, Theorem5], $A^{\perp} \quad$ is a semiprime ideal. Thus by corollary 2.5 ( $i i)$ holds.

$(i i) \Rightarrow(i)$. Conversely, let (ii) holds but $\mathrm{S}$ is not 0 -distributive. Then there exists $a, b, c \in S$ with $a \wedge b=0=a \wedge c$ and $b \vee c$ exists but $a \wedge(b \vee c) \neq 0$. Set $F=\{x \in S: x \geq a \wedge(b \vee c)\}$. Then $F$ is a proper filter as $0 \notin F$. Also $a \in F$ and $b \vee c \in F$. But for any $x \in F, a \wedge x \geq a \wedge(b \vee c) \neq 0$. implies $x \notin A^{\perp}$. Therefore, $F \cap A^{\perp}=\phi$. Hence by ( $\left.i i\right)$, there exists a prime filter $Q \supseteq F$ such that $Q \cap A^{\perp}=\phi$ . Then $b \vee c \notin S-Q$. This implies either $b \notin S-Q$ or $c \notin S-Q$ as $S-Q$ is a prime ideal. Hence either $b \in Q$ or $c \in Q$. Therefore, either $a \wedge b \in Q$ or $a \wedge c \in Q$. Which implies $0 \in Q$ and this gives a contradiction that $Q$ is a prime filter. Hence $S$ must be 0 -distributive.

Acknowledgement. Before conclusion, we would like to pay our sincere gratitude and thanks to the honorable reviewer for his valuable criticism, remarks and comments for the fulfillment of the paper.

\section{Conclusion}

Glivenko congruence is an important topic distributive lattices. This concept has been extended for 0-distributive lattices. In this paper we have generalized the concept for 0-distributive nearlattices, which are of course non distributive nearlattices. In future, extending the results of this paper futher research can be down for general non distributive nearlattices with 0 .

\section{REFERENCES}

1. M.Ayub Ali, R.M.Hafizur Rahman, A.S.A.noor and M.M.Rahman, Some properties of semi prime ideals in a lattice, Annals of Pure and Applied Mathematics, 1(2) (2012) 176-185.

2. P.Balasubramani and P.V.Venkatanarasimhan, Characterizations of the 0distributive lattice, Indian J. Pure Appl. Math., 32(3) (2001) 315-324.

3. A.S.A.Noor and M.B.Rahman, Separation properties in nearlattices, The Rajshahi University Studies (Part B) 22(1994) 181-188.

4. A.S.A.Noor and M.Begum, Some properties of 0-distributive meet semilattices, Annals of Pure and Applied Mathematics, 2(1) (2012) 60-66.

5. A.S.A.Noor, Md. Zaidur Rahman and Md. Bazlar Rahman, Some characterization of 0-distributive nearlattice, Annals of Pure and Applied Mathematics, 2(2) (2012) 194199.

6. Y.S.Pawar and N.K.Thakare, 0-distributive semilattices, Canad. Math. Bull., 21(4) (1978) 469-475.

7. Y.Rav, Semi prime ideals in general lattices, Journal of Pure and Applied Algebra, 56 (1989) 105-118. 
Glivenko Congruence on a Nearlattice Related to Semi Prime Ideals

8. J.C.Varlet, A generalization of the notion of pseudo-complementedness, Bull.Soc. Sci Liege, 37(1968) 149-158.

9. Md. Zaidur Rahman, Md. Bazlar Rahman and A.S.A.Noor, 0-distributive nearlattice, Annals of Pure and Applied Mathematics, 2(2) (2012) 177-184.

10. Md Zaidur Rahman, Md Bazlar Rahman and A.S.A.Noor, Some properties of 0distributive nearlattice, Annals of Pure and Applied Mathematics, 2(2) (2012) 185193.

11. Md Zaidur Rahman, Md Bazlar Rahman and A.S.A.Noor, On semi prime ideals in nearlattices, Annals of Pure and Applied Mathematics, 3(1) (2012) 1- 9.

12. A.S.A.Noor and Md. Zaidur Rahman, On Glivenko congruence of a 0-distributive nearlattices, Annals of Pure and Applied Mathematics, 3(2) (2013) 108-112. 